
Сибирский математический журнал

Ноябрь-декабрь, 2019. Том 60, № 6

УДК $510.223+510.225$

\title{
АБСОЛЮТНОСТЬ МНОЖЕСТВА $\Sigma$ ПО СОЛОВЕЮ
}

\section{В. Г. Кановей, В. А. Любецкий}

\begin{abstract}
Аннотация. Доказано, что множество $\Sigma$ по Соловею в достаточно широком смысле абсолютно определимо, в частности, не зависит от выбора исходной модели.
\end{abstract}

DOI 10.33048/smzh.2019.60.608

Ключевые слова: абсолютность, генеричность, множество $\Sigma$ по Соловею.

\section{1. Введение}

Главный результат классической статьи Соловея [1] состоит в построении указанной в ее названии модели и второй модели, в которой измеримыми являются только определимые множества, но аксиома выбора верна в отличие от первой модели, где она нарушена. Ключевую роль в построении этих моделей выполняла «важная лемма» из [1, разд. 4.4], согласно которой любое генерическое расширение исходной модели является генерическим расширением и любой промежуточной модели.

Точнее говоря, если $\mathbb{P}=\langle\mathbb{P} ; \leqslant\rangle^{1)}$ - форсинг в теоретико-множественном универсуме $\mathbf{V}, t \in \mathbf{V}$ есть простое $\mathbb{P}$-имя (т. е. $t \subseteq \mathbb{P} \times \mathbf{V}$ ), множество $G \subseteq \mathbb{P}$

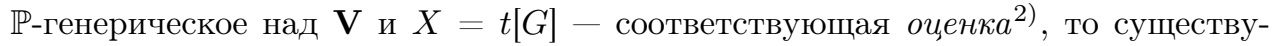
ет множество $\Sigma=\Sigma_{\mathbb{P} t}^{\mathbf{V}}(X) \subseteq \mathbb{P}$, для которого $\mathbf{V}[\Sigma]=\mathbf{V}[X], G \subseteq \Sigma$ и $G \Sigma$ генерическое над $\mathbf{V}[X]$. Таким образом, $\mathbf{V}[G]$ есть $\Sigma$-генерическое расширение промежуточной модели $\mathbf{V}[X] \subseteq \mathbf{V}[G]$.

Впоследствии строение промежуточных моделей рассматривалось в работах [2-6] и др., а также в [7], где установлено, что в указанных условиях и само множество $\Sigma=\Sigma \Sigma_{\mathbb{P} t}^{\mathbf{V}}(X)$ генерическое над $\mathbf{V}$ в смысле форсинга $\mathbb{P}_{t}=\langle\mathbb{P} ; \leqslant t\rangle$ с той же областью $\mathbb{P}$ и с отношением порядка $\leqslant_{t}=\leq_{\mathbb{P} t}$, которое $\subseteq$-расширяет данный порядок $\leqslant$. Таким образом, сама промежуточная модель $\mathbf{V}[X]=\mathbf{V}[\Sigma]$ становится генерическим расширением данного универсума $\mathbf{V}$.

Исследования последних лет $[8,9]$ показали, что в работе со сложными итерированными генерическими расширениями важную роль играет абсолютность определения множества $\Sigma_{\mathbb{P} t}^{\mathbb{V}}(X) \subseteq \mathbb{P}$ и порядка $\leq \mathbb{P} t$ в плане независимости от универсума $\mathbf{V}$. (Зависимость от $\mathbb{P}, t, X$ очевидна и неустранима.) Эта независимость установлена в [9] ad hoc в одном частном случае. Здесь доказывается общий результат.

Работа выполнена при финансовой поддержке Российского фонда фундаментальных исследований, гранты РФФИ 17-01-00705 (В. Г. Кановей), РФФИ 18-29-13037 (В. А. Любецкий).

1) $p \leqslant q$ означает, что вынуждающее условие $q$ более сильное, чем $p$.

${ }^{2)} t[G]=\{x: \exists p \in G(\langle p, x\rangle \in t)\} \in \mathbf{V}[G]$ есть $G$-оченка для $t ; t[G] \subseteq \mathbf{V}$.

(c) 2019 Кановей В. Г., Любецкий В. А. 
Теорема 1. Пусть $\mathbb{P}=\langle\mathbb{P} ; \leqslant\rangle \in \mathbf{V}-$ форсинг в универсуме $\mathbf{V}, t \in \mathbf{V}$ есть $\mathbb{P}$-имя, множество $G \subseteq \mathbb{P} \mathbb{P}$-генерическое над $\mathbf{V}, X=t[G] \subseteq \mathbf{V}$. Пусть дополнительно $\mathbf{V}-$ генерическое расширение класса $\mathbf{L}[\mathbb{P}, t] \subseteq \mathbf{V}$. Тогда $\Sigma_{\mathbb{P} t}^{\mathbf{V}}(X)=$ $\Sigma_{\mathbb{P} t}^{\mathbf{L}[\mathbb{P}, t]}(X)$, а отношение $\leq_{\mathbb{P} t}^{\mathbf{V}}$ тождественно $\leq_{\mathbb{P} t}^{\mathbf{L}[\mathbb{P}, t]}$.

Эта теорема находит применение в исследовании промежуточных моделей расширений, генерических в смысле «случайного» форсинга Соловея [10], вообще одного из наиболее широко применяемых форсингов в современной теории множеств $^{3)}$, и может найти применения к промежуточным моделям других генерических расширений таких, как, например, генерические расширения, рассмотренные в $[13,14]$.

\section{2. Множество Соловея $\Sigma$ и отношение $\leqslant_{t}$}

Определение множества $\Sigma=\Sigma_{\mathbb{P} t}^{\mathrm{V}}(X)$ по [1] состоит в следующем.

ОПРедЕЛЕниЕ 2 (в условиях теоремы 1 ). Полагаем $\Sigma=\Sigma_{\mathbb{P} t}^{\mathbb{V}}(X)==_{\text {def }} \mathbb{P} \backslash$ $\bigcup_{\xi<\vartheta} A_{\xi}$, где ординал $\vartheta$ определен в ходе построения, а последовательность множеств $A_{\xi}=A_{\xi}^{\mathrm{V}} \subseteq \mathbb{P}$ определяется в $\mathrm{V}[X]$ по индукции:

(1) $A_{0}$ состоит из всех «условий» $p \in \mathbb{P}$, вынуждающих $\breve{x} \in t$ для некоторого $x \in \mathbf{V} \backslash X$, или $\breve{x} \notin t$ для некоторого $x \in X^{4)}$.

(2) $A_{\xi+1}$ состоит из всех таких «условий» $p \in \mathbb{P}$, для которых существует плотное множество $D \in \mathbf{V}$ в $\mathbb{P}$, удовлетворяющее следующему: если $q \in D$ и $p \leq q$, то $q \in A_{\xi}$.

(3) $A_{\lambda}=\bigcup_{\xi<\lambda} A_{\xi}$ для предельных ординалов $\lambda$.

Итак, каждое «условие» $p \in A_{0}$ прямо противоречит допущению, что $t-$ имя для $X$, по (1), и это противоречие сохраняется пунктами (2) и (3) индуктивного определения для всё больших индексов $\xi$, соответственно во всё более непрямой форме. Эта $\subseteq$-возрастающая последовательность множеств $A_{\xi} \subseteq \mathbb{P}$ стабилизируется на каком-то предельном ординале $\vartheta \in \mathbf{V}$, и имеем множества $A=\bigcup_{\xi<\vartheta} A_{\xi}$ и $\Sigma=\mathbb{P} \backslash A$.

Отношение $\leqslant_{t}=\leq_{\mathbb{P} t}^{\mathrm{V}}$, второй предмет нашего интереса, вводится так.

ОПРЕДЕЛЕНИЕ 3 ([7], в условиях теоремы 1 ). Если $p, q \in \mathbb{P}$, то $p \leqslant_{t} q$ определяется, когда «условие» $q \mathbb{P}$-вынуждает над $\mathbf{V}$, что $\breve{p} \in \Sigma_{\breve{\mathbb{P}}, \breve{t}}^{\breve{t}}(\breve{t}[\underline{G}])$.

Другими словами, для $p \leq \mathbb{P} t q$ необходимо и достаточно, чтобы соотношение $p \in \Sigma_{\mathbb{P} t}^{\mathbf{V}}(X)$ выполнялось всякий раз, когда множество $G \subseteq \mathbb{P}$ генерическое над $\mathbf{V}, X=t[G]$ и $q \in G$.

Следующие два результата выражают ключевые свойства множеств $\Sigma_{\mathbb{P} t}^{\mathrm{V}}(X)$ и отношений $\leq \mathbb{P} t$ в контексте форсинга. Они не используются в доказательстве теоремы 1 и приведены только из соображений полноты изложения.

Предложение 4 (см. [1, 4.4] или [15, 13.3.2]). В обозначениях определения 2 множество $\Sigma=\Sigma_{\mathbb{P} t}^{\mathrm{V}}(X)$ удовлетворяет таким условиям:

\footnotetext{
${ }^{3)} \mathrm{Cм.,} \mathrm{например,} \mathrm{нашу} \mathrm{статью} \mathrm{[11]} \mathrm{о} \mathrm{некоторых} \mathrm{приложениях} \mathrm{«случайного»} \mathrm{форсинга}$ Соловея. Избегая дальнейших ссылок, упомянем только так и не опубликованное исследование Е. И. Гордона [12], где случайный форсинг использован для доказательства нескольких теорем о продолжении мер Хаара.

4) Если $x \in \mathbf{V}$, то $\breve{x}=\mathbb{P} \times x-$ каноническое имя для $x$.
} 
(i) $\Sigma$ замкнуто вниз в $\mathbb{P}$, т. е. если $q \in \Sigma, p \in \mathbb{P}$ и $p \leq q$, то $p \in \Sigma$;

(ii) $\mathbf{V}[\Sigma]=\mathbf{V}[X]$;

(iii) $G \subseteq \Sigma$ и $G \Sigma$-генерическое над $\mathbf{V}[X]$, следовательно, $\mathbf{V}[G]$ есть $\Sigma$ генерическое расширение модели $\mathbf{V}[X]=\mathbf{V}[\Sigma]$;

(iv) если множество $G^{\prime} \subseteq \Sigma \Sigma$-генерическое над $\mathbf{V}[X]$ то оно также $\mathbb{P}$ генерическое над $\mathbf{V}$ и, сверх того, $t\left[G^{\prime}\right]=X$.

Предложение 5 (см. [7]). (i) $\leq \mathbb{P} t$ есть отношение частичного порядка на $\mathbb{P}$. Оно принадлежит $\mathbf{V}$ и продолжает данный порядок $\leqslant=\leqslant \mathbb{P}$, т. $е . \leqslant \subseteq \leq \mathbb{P} t$ (или, равносильно, соотношение $p \leqslant q$ влечет $p \leq \mathbb{\mathbb { P } t} q$ ).

(ii) Если множество $G \subseteq \mathbb{P}$ генерическое над $\mathbf{V}$ и $X=t[G]$, то множество $\Sigma=\Sigma_{\mathbb{P} t}^{\mathbf{V}}(X)$ генерическое над $\mathbf{V}$ в смысле форсинга $\langle\mathbb{P} ; \leq \mathbb{P} t\rangle$.

\section{3. Абсолютность множества $\Sigma$ и отношения $\leqslant_{t}$}

ДокаЗАТЕЛЬСТво теОРемЫ 1. ЧАСть 1. Докажем, что выполнено равенство $\Sigma_{\mathbb{P} t}^{\mathrm{V}}(X)=\Sigma_{\mathbb{P} t}^{\mathbf{L}}[\mathbb{P}, t](X)$. По условию найдутся форсинг $Q=\left\langle Q ; \leq_{Q}\right\rangle \in \mathbf{L}[\mathbb{P}, t]$ и $Q$-генерическое над $\mathbf{L}[\mathbb{P}, t]$ множество $H \subseteq Q$, удовлетворяющее $\mathbf{V}=\mathbf{L}[\mathbb{P}, t][H]$. Напомним, что по условию множество $G \mathbb{P}$-генерическое над $\mathbf{V}$, откуда по теореме о произведении форсингов следует, что $H Q$-генерическое и над $\mathbf{L}[\mathbb{P}, t][G]$.

Возвращаясь к определению 2 , докажем по индукции, что $A_{\xi}^{\mathbf{V}}=A_{\xi}^{\mathbf{L}[\mathbb{P}, t]}$.

Достаточно рассмотреть индуктивный шаг $\xi \rightarrow \xi+1$ п. (2) определения 2. Таким образом, пусть $A_{\xi}^{\mathrm{V}}=A_{\xi}^{\mathrm{L}[\mathbb{P}, t]}=A_{\xi}$, и требуется доказать, что $A_{\xi+1}^{\mathrm{V}}=$ $A_{\xi+1}^{\mathbf{L}[\mathbb{P}, t]}$. Коль скоро $\mathbf{L}[\mathbb{P}, t] \subseteq \mathbf{V}$, имеем $A_{\xi+1}^{\mathbf{L}[\mathbb{P}, t]} \subseteq A_{\xi+1}^{\mathbf{V}}$. Для вывода обратного включения допустим, что $p_{0} \in A_{\xi+1}^{\mathbf{V}}$ через посредство плотного множества $D \in$ $\mathbf{V}, D \subseteq \mathbb{P}$, как в п. (2) определения 2. Требуется вывести $p_{0} \in A_{\xi+1}^{\mathrm{L}[\mathbb{P}, t]}$.

Имеем $D=\tau[H]$, где $\tau \in \mathbf{L}[\mathbb{P}, t], \tau \subseteq Q \times \mathbb{P}$ (подходящее простое $Q$-имя для подмножества $\mathbb{P})$. Не ограничивая общности, предполагаем, что

$$
\left(\langle q, p\rangle \in \tau \wedge q^{\prime} \in Q \wedge q \leq_{Q} q^{\prime}\right) \Longrightarrow\left\langle q^{\prime}, p\right\rangle \in \tau .
$$

Имеется «условие» $q_{0} \in H, Q$-вынуждающее

$$
\tau[\underline{H}] \text { плотное } \wedge \forall p \in \tau[\underline{H}]\left(p_{0} \leqslant p \Longrightarrow p \in A_{\xi}^{\smile}\right)
$$

над $\mathbf{L}[\mathbb{P}, t][X]$, где $A_{\xi}^{\smile}=Q \times A_{\xi}$, как обычно, каноническое $Q$-имя для множества $A_{\xi} \in \mathbf{L}[\mathbb{P}, t][X]$. Рассмотрим множества

$$
D_{1}^{\prime}=\left\{p \in \mathbb{P}: p_{0} \leqslant p \wedge \exists q \in Q\left(q_{0} \leq_{Q} q \wedge\langle q, p\rangle \in \tau\right)\right\}
$$

и $D_{2}^{\prime}=\left\{p \in \mathbb{P}: p_{0}, p \text { несовместны в } \mathbb{P}\right\}^{5)}$. Понятно, что эти множества принадлежат $\mathbf{L}[\mathbb{P}, t]$, как и их объединение $D^{\prime}=D_{1}^{\prime} \cup D_{2}^{\prime}$.

Утверждаем, что $D^{\prime}$ плотно в $\mathbb{P}$. В самом деле, пусть $p \in \mathbb{P}$. Если $p$ несовместно с $p_{0}$, то сразу $p \in D^{\prime}$. Если $p$ совместно с $p_{0}$, то можно предполагать, что $p_{0} \leqslant p$. Поскольку $q_{0}$ вынуждает $(\dagger)$, имеются такое «условие» $q \in H$, что $q_{0} \leq_{Q} q$, и также некоторое $p^{\prime} \in \mathbb{P}$, для которого $p \leqslant p^{\prime}$ и $q$ вынуждает $p^{\prime} \in \tau[\underline{H}]$. Тогда $\left\langle q, p^{\prime}\right\rangle \in \tau$ согласно $(*)$ и $p^{\prime} \in D^{\prime}$, что и требовалось.

Также утверждаем, что если $p \in D^{\prime}$ и $p_{0} \leqslant p$, то $p \in A_{\xi}$. В самом деле, $p \notin$ $D_{2}^{\prime}$, поэтому $p \in D_{1}^{\prime}$ посредством какого-то «условия» $q \in Q$, так что $q_{0} \leq_{Q} q$ и

\footnotetext{
5) Несовместность означает, что нет ни одного $p^{\prime} \in \mathbb{P}$, для которого $p_{0} \leqslant p^{\prime}$ и $p \leqslant p^{\prime}$.
} 
$\langle q, p\rangle \in \tau$. Тогда $q$ вынуждает $p \in \tau[\underline{H}]$, следовательно, коль скоро $q$ вынуждает и $(\dagger)$, заключаем, что $p \in A_{\xi}$, что и требовалось.

По доказанному множество $D^{\prime}$ гарантирует, что $p_{0} \in A_{\xi+1}^{\mathbf{L}[\mathbb{P}, t]}$, завершая индуктивный шаг.

ЧАСть 2. Докажем, что отношение $\leq_{\mathbb{P} t}^{\mathrm{V}}$ тождественно $\leq_{\mathbb{P} t}^{\mathbf{L}[\mathbb{P}, t]}$. Пусть $p, q \in$ $\mathbb{P}$. Требуется доказать, что следующие два соотношения эквивалентны:

(А) если множество $G \subseteq \mathbb{P} \mathbb{P}$-генерическое над $\mathbf{V}, X=t[G]$ и $q \in G$, то $p \in \Sigma_{\mathbb{P} t}^{\mathrm{V}}(X)$

(В) если множество $G \subseteq \mathbb{P} \mathbb{P}$-генерическое над $\mathbf{L}[\mathbb{P}, t], X=t[G]$ и $q \in G$, то $p \in \Sigma_{\mathbb{P} t}^{\mathbf{L}}[\mathbb{P}, t](X)$.

При этом импликация $(\mathrm{A}) \Longrightarrow(\mathrm{B})$ следует из уже доказанного равенства $\Sigma_{\mathbb{P} t}^{\mathbf{V}}(X)=\Sigma_{\mathbb{P} t}^{\mathbf{L}[\mathbb{P}, t]}(X)$, поскольку всякое множество, генерическое над $\mathbf{V}$, будет генерическим и над меньшей моделью $\mathbf{L}[\mathbb{P}, t] \subseteq \mathbf{V}$.

Для доказательства обратной импликации $(\mathrm{B}) \Longrightarrow(\mathrm{A})$ предположим, что (А) не выполнено. Это вынуждается каким-то «условием» $r \in G, r \geq q$, т. е. имеем следующее:

(C) если множество $G \subseteq \mathbb{P}$ генерическое над $\mathbf{V}, X=t[G]$ и $r \in G$, то $p \notin \Sigma_{\mathbb{P} t}^{\mathbb{V}}(X)$.

Убедимся, что тогда и (В) не выполняется. Для этого рассмотрим произвольное множество $G \subseteq \mathbb{P}, \mathbb{P}$-генерическое над $\mathbf{V}$ и содержащее $r$. Пусть $X=t[G]$. Тогда $p \notin \Sigma_{\mathbb{P} t}^{\mathrm{V}}(X)$ согласно $(\mathrm{C})$, следовательно, $p \notin \Sigma_{\mathbb{P} t}^{\mathbf{L}}[\mathbb{P}, t](X)$ по доказанному выше. Но $q$ также принадлежит $G$, поскольку $q \leqslant p$. Значит, (В) не выполняется, что и требовалось.

\section{4. Вопросы}

Boпрос 6. Посылка теоремы 1 о том, что данный универсум V есть генерическое расширение своего подкласса $\mathbf{L}[\mathbb{P}, t]$, является необходимым элементом нашего доказательства в той его части, которая связана с множеством $D^{\prime}$. Bepна ли теорема 1 без этой посылки о генеричности универсума $\mathbf{V}$ ?

Вопрос 7. Было бы интересно получить аналог нашей теоремы абсолютности в терминах булево-алгебраической версии форсинга. Отметим в этой связи следующее. Шаг от промежуточной модели $\mathbf{V}[X]$ к общему расширению $\mathbf{V}[G]$ в принципе хорошо изучен в булево-алгебраической версии и сводится к замене множества $\Sigma$ по Соловею некоторой подалгеброй той ПБА, в которую канонически вкладывается форсинг $\mathbb{P}$ (см., к примеру, [16, лемма 69]). В то же время шаг от исходной модели $\mathbf{V}$ к промежуточному расширению $\mathbf{V}[X]$ в этом плане изучен недостаточно, в особенности в контексте нашего результата из [7] о генеричности множества $\Sigma$ в смысле измененного порядка $\leqslant_{t}$. Также важно, что полные булевы алгебры сами по себе не абсолютны при переходе от одной модели к другой, как, к примеру, множество $\mathbb{R}$ вещественных чисел не абсолютно при добавлении нового числа к исходной модели. На данный момент нам неясно, насколько эти трудности преодолимы на пути к булево-алгебраической форме нашей теоремы 1.

Авторы признательны анонимному рецензенту за ценные замечания, позволившие дополнить и улучшить изложение, в частности, добавить вопрос 7. 


\section{ЛИТЕРАТУРА}

1. Solovay R. M. A model of set theory in which every set of reals is Lebesgue measurable // Ann. Math. 1970. V. 92. P. 1-56.

2. Grigorieff S. Intermediate submodels and generic extensions of set theory // Ann. Math. 1975. V. 101. P. 447-490.

3. Zapletal J. Terminal notions in set theory // Ann. Pure Appl. Logic. 2001. V. 109, N 1-2. P. 89-116.

4. Kanamori A. The higher infinite. Large cardinals in set theory from their beginnings. Berlin: Springer-Verl., 2003.

5. Jech T. Set theory. The third millennium revised and expanded. Berlin: Springer-Verl., 2003.

6. Кановей В. Г., Любецкий В. А. Обобщение одной конструкции Соловея // Сиб. мат. журн. 2015. Т. 56, № 6. С. 1341-1350.

7. Кановей В. Г., Любецкий В. А. Генерическое свойство множества $\Sigma$ по Соловею // Сиб. мат. журн. 2017. Т. 58, № 6. С. 1302-1305.

8. Fuchs G., Hamkins J. D., Reitz J. Set-theoretic geology // Ann. Pure Appl. Logic. 2015. V. 166, N 4. P. 464-501.

9. Kanovei V., Lyubetsky V. On Harrington's model in which Separation holds but Reduction fails at the 3rd projective level, and on some related models of Sami. 2018. 21 p. Eprint arXiv 1810.12542.

10. Kanovei $V$., Lyubetsky $V$. On intermediate extensions of generic extensions by a random real. 2018. 11 p. arXiv 1811.10568.

11. Кановей В. Г., Любецкий В. А. О некоторых классических проблемах дескриптивной теории множеств // Успехи мат. наук. 2003. Т. 58, № 5. С. 3-88.

12. Гордон Е. И. 0 продолжении мера Хаара в сигма-компактных группах. 1981. 26 с. Деп. в ВИНИТИ. № 1244-81.

13. Кановей В. Г., Любецкий В. А. Эффективная минимальная кодировка несчетных множеств // Сиб. мат. журн. 2011. Т. 52, № 5. С. 1074-1086.

14. Kanovei $V$. Non-Glimm-Effros equivalence relations at second projective level // Fundam. Math. 1997. V. 154, N 1. P. 1-35.

15. Кановей В. Г., Любецкий В. А. Современная теория множеств: абсолютно неразрешимые классические проблемы. М.: МЦНМО, 2013

16. Йех Т. Теория множеств и метод форсинга. М.: Мир, 1973.

Поступила в редакиию 19 декабря 2018 г.

После доработки 10 апреля 2019 г.

Принята к публикащии 15 мая 2019 г.

Кановей Владимир Григорьевич, Любецкий Владимир Александрович

Институт проблем передачи информации им. А. А. Харкевича РАН,

Большой Каретный переулок, 19, стр. 1, Москва 127051

kanovei@iitp.ru (автор для контактов), lyubetsk@iitp.ru 